\title{
TWENTY-FIVE YEARS OF ORAL RECONSTRUCTION
}

BIOMET 3i, a world leader in oral reconstructive devices, is celebrating its 25th anniversary. Founded in 1987 as Implant Innovations Inc. ('3i') by a periodontist and an engineer, the company has grown to 1,000 employees with its global headquarters located in Palm Beach Gardens, Florida.

BIOMET 3i has won worldwide acclaim for the microtextured surface of the OSSEOTITE Implant, which has more than 15 years of documented research. More recently, BIOMET 3i

introduced a Bone Bonding NanoTite Surface and has also been recognised for its contributions to new dental technologies, such as digital dentistry, with the development of its patented BellaTek Encode Impression System allowing for a quicker, more comfortable impression process for the patient.

For further information on BIOMET 3i products, call 01628519162 or email 3iukm.marketing@biomet.com.

\section{EXACT SHADE MATCHING GUARANTEED}

Vitique is DMG's newest composite luting cement for veneers, crowns, bridges, inlays and onlays. It offers both light curing and dual curing facilities.

Vitique can be applied directly using DMG's patented Cata-Lock syringe application system. Simply by clipping the catalyst syringe onto the base syringe, the light curing system is turned into a dual curing system. This assembly combines the syringes so that both pastes are automatically dosed and mixed in the optimised proportions. As only a small amount of catalyst is used, minimal shade change occurs, thus ensuring consistent shade matching. Exact shade matching is guaranteed every time.

When cementing veneers, the Veneer-Tip dispenses a 'ribbon' of base material onto the fitting surface of the veneer. When using the base/ catalyst combination, mixed cement is dispensed onto the fitting surface via a conventional mixing tip.

Radiopaque, Vitique consists of eight base shades, corresponding try-in pastes, two catalyst consistencies and a try-in paste in the catalyst shade. It is available in an Introductory Kit and Refill Packs of all the individual components.

For further information call 01656 789401, emailinfo@dmg-dental.co.uk or visit www.dmg-dental.com.

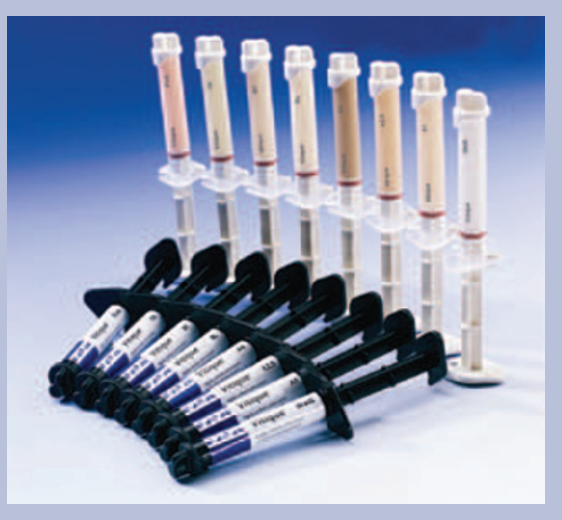

\section{STEERING YOUR DENTAL TEAM TO SUCCESS}

To help you make the best financial decisions, specialist Independent Financial Adviser money4dentists can help advise you on ways to make the most efficient use of your money.

With over half a century of experience providing high quality financial advice to dentists, money4dentists can offer guidance and support on anything from pensions and tax to debt management, investments and savings. money4dentists is particularly aware of the unique challenges facing members of the dental team, and offers bespoke financial advice specifically tailored to the dental profession. Whatever your financial concerns, money4dentists' expert team are on-hand to help your steer a course to financial success.

For more information please 0845 345 5060, emailinfo@money4dentists. com or visit www.money4dentists.com. 